\title{
Evolution at the Surface of Euclid: Elements of A Long Infinity In Motion Along Space
}

Marvin E. Kirsh, California State University Los Angeles US

Keywords: mind and matter, social and natural evolution, discourse and power, energy and form

\begin{abstract}
It is modernly debated whether application of the free will has potential to cause harm to nature. Power possessed to the discourse, sensory/perceptual, physical influences on life experience by the slow moving machinery of change is a viral element in the problems of civilization; failed resolution of historical paradox involving mind and matter is a recurring source of problems. Reference is taken from the writing of Euclid in which a oneness of nature as an indivisible point of thought is made prerequisite in criteria of interpretation to demonstrate that contemporary scientific methodologies alternately ensue from the point of empirically centered induction. A qualification for the conceptualization is proposed that involves a physically describable form bound to energy in addition to contemporary notions of energy bound to form and a visually based mathematical-physical form is elaborated and discussed with respect to biological and natural processes.
\end{abstract}

Keywords: energy matter and geometrical form, The Elements of Euclid, science discourse and power, mind and matter, the concept

\section{Introduction}

". This then is demonstrable, but it is an axiom that every continuum is divisible; hence a finite line, being continuous, is divisible. This is the notion that the author of the Elements uses in bisecting the finite straight line, and not the assumption that it is divisible to infinity. That something is divisible and that it is divisible to infinity are not the same thing." (Morrow, 1970a).

The philosophy of Logical Positivism (Karnap, 1956) of the twentieth century proposes as safe criteria for the validity of scientific theory an alignment of induced, philosophically found interpretation with empirical test. If real paths of natural progress are necessarily rendered from this methodology, it presumes either that a single unique path of nature exists, or that all paths established by empirical method are relevant for direct focus in application to problems. The philosophy of Post Modernism (Greer, 2003) in a further addition purports that truth is a creation of path and is not discovered. The philosophies of Logical Positivism and Post 
Modernism philosophies mutually rely strictly on a notion that all trajectories possess an equal qualitative and quantitative open direction for the future. Rorty (Tartaglia, 2007) has added the opinion that men are free to apply their free will to the elements of nature without fear that they can damage it.

Though it can be argued that if a point of reference exists it is in open, perceived to be infinite space, that 'infinite' has qualitative meaning only and all open spaces are not equal; death may be considered to represent the closed state, obviously all entities do not have the same lifetime. The existence of any path entails only relative degrees of openness, either a line or a circle, extrapolated from its' existence on plane (excuse the pun but paper is plain) paper to open space can be envisioned to represent varying degrees openness. It is obviously the discrete point of thought only that can be sufficiently and validly extrapolated from the plane of its' existence to open space; for potential validity, the intersection of intuitive induction and reality can be centered only from the first and most enduring point of thought, of uniqueness, and identity possessed to all of the elements of experience; 'oneness' other than plurality must be the criteria must be of interpretation, other to deny identity as a criteria for existence, to deny existence. Euclid interpreted the circle a motion of the discrete point around a center to be the limit of all things. In this presentation, the circle is taken as a discrete point and an egg shaped space as a point of thought is evolved mathematically as a motion of the circle along a straight divisible line that is meant to represent a maximally open path. It is suggested that modern science methodologies, the philosophies of Logical Positivism and Post Modernism assume in point of thought, other than the maximum open of a straight line, curved or ultimately circularly closed paths meant to encompass the notion of oneness as the point of thought in conceptualizations.

\section{Discussion}

It cannot be denied in argument that the concept 'universe' entails the concept 'path' and visa versa; knowledge has a path, 'path' entails both the concept of path and from perceptual reference, parametric specificity. There is no type of knowledge that can exclude a familiarity with living, living a familiarity with change which has no other means of communication than by the entailment of path. All concepts originate from the motions of interactions that again entail a temporal path. If the word 'universe' is restricted to mean all that which life experiences and the senses can refer; 'path' refers to the 'universe', 'universe' refers to 'path'. Remaining to the imagination, at the borderlines between science and life experience are still cases of path, of the universe as a statistical entity, or as a set of historical names-i.e. "a universe" or "The Universe"," A path" or "The path". In correspondence, a division of invention and nature the productions of cognition and that which exists in nature, can be witnessed in Euclid's creation of 'The Elements" (Proclus, 1970): Euclid's dialogue, originating at the indivisible point of intersection of mathematics and philosophy, maintains the continuity of an infinite line that originates from an indivisible point of philosophy and not mathematical invention, is given, by rule, to possess dividable connections from one element to the next as if fearing to tread into the uncharted, if not unknowable, waters of the constructions of nature that yield experience of it, and not on points of philosophical argument; invention as substitute for the natural, as if profane, is avoided, the works of nature are not considered works of art. Whether postmodern philosophies posit truth away from the category of unmasking, to the category of its' creation 
as the work of nature, either the result of the application of impulse, 'path' is ubiquitously entailed as either a path to its' revelation, or to or from its' creation: within the common perspective, either publicly put to test is the body of scientific knowledge or in private its' soul, i.e.-the means of rational comprehension of the happenstances from which the path towards tomorrow can proceed. The topic of change as a manifestation of power or as the power of manifestation has not escaped the active gaze of judgment of those who see to court it; in doing so engender power itself to the same discourse that contains it (Foucault, 1977).

In a universe postulated to be composed of occurrences possessing a unique, distinct, location and place dependent concept of time for events, rather than a universe that possesses time relating to a unique, single, ubiquitous and contiguous process, to a unique path, but to time as a concept only whose relative measure with respect to events in which the notion of simultaneity is logically incoherent, logically referred is the necessity for a witnessibility of events and a first person perspective for description. If description of both historical events and a construction of the path of processes evolve from a co-evolution of the physical and conceptual, temporal paralleling and subsequent aligning of the physical and conceptual can be proposed to occur upon the achievement of knowledge, then the conceptual and physical, memory and path, the immaterial and the material, respectively, are the effect of emerging path that is not describable with universal numerical constants, but change with the perspective dependant means of the elaboration of time. The universe, argued to be contained in definition as the effect of change, can be descriptively embodied from a scientific viewpoint as a perspective dependent path of propagation at any point of witness referral as the reflection or witness of unwitnessibile energy that is defined as a state of constantly becoming witnessible matter: the concept can be encompassed as an unwitnessible difference state of witnessible or reflected energy, becomingwitnessibile matter. The universe in this description is divided between the witnessible and unwitnessible to possess strictly unwitnessible energy that is conceptual and the material products of its' witness.

In this presentation, to elaborate a universal form, a form for energy is derived and is added to modern cause and effect conceptualizations that in converse dwell singularly on an association of energy with form. Constantly becoming products of interactions of physical volumes of space can be envisioned to parallel the witness-pair dependant distribution of formed concepts in learning processes whose evolution is also a perspective dependent matter that is evolved from proximal relations of memory that are ultimately a product of physical volumes that form the environment; 'The concept' is conceived to be included in universal description as a uniquely existing but distinctly evolving parallel to uniquely existing physical spaces as in a nominalists' view of the world; it is accommodated within a scientific framework with the property of unwitnessibility. The evolution of witnessible sensory experience is held as the only criteria for the existence of volume appropriate to tangible witness; the existence of an unwitnessible sort of volume/energy-state can emerge from the point of witness perspective in which its' characterization involves an unwitnessible displacement of some sort as a difference ratio of the materially witnessed as they are evolved from unreflected, unwitnessible to the senses until reflected, energies (i.e. the concept of color can be taken to be the result of a synergy of energies of proximal radiations of witnessed energy of various colors). For 
explanation as a universal holism, a universal consciousness, 'the concept', as a difference state of reflected energies, is an unwitnessible ubiquitous property of all spaces to include those processes responsible for both physiological and cognitive functioning, the internal energetic processes and motions associated with the life process, as well as physical motion of the entity, processes of particular environments. Cognitive interpretation, perception, sensory experience are given simultaneous existence and explanation with the lengths and motions that are external to the entity; all the miniature to the vast, internal and external is reduced conceptually to be the product of differences, synergies, of the products of the unwitnessible-in advance-of-witness with the associated meaning that 'unwitnessible' entails a non-coherent meaning to the element of time, time as a concept becomes realizable only upon the event of witness and possesses numerical potential only as a measure that is place-of-event dependent.. Place-of-event dependency is meant to refer to the location dependent diversity and unique identity of events. Simultaneity is here translocated from the meaning given in relativity theory to refer to simultaneity as a mathematical relativity of time, to be absent of time and to only indicate a universal associated with the event of witness as it underlines all processes.

The notion of 'concept' entailing ordering of the elements experience for the purpose of memory associated learning For this purpose a relative arc length of an angle to the line between witnesses in witness pair relations can be used to lend temporal priorities to concepts that are dependent on the parameters of a situation (Figure 3). Combined physical and conceptual reality is associated with a dynamic state involving the intersection of proximally inherited associated states. Language as the sum of learned/acquired conceptual states can be captured to the cumulative composite of a dynamically changing plurality of angles of arcs that are actively organized into hierarchies of priorities whose order in terms of appropriate application are dependent on the particulars of the witness-environment encounter and association. The concept is construed to possess more of an absolute value than environmental energy parameters conversely drawn from a slower rate of change, constant state of the perceived environment, and reflects a scale of magnitudes of importance in relation to other concepts that in turn is a creation of the particulars of surface terrains that contain the witness event. Ideation of an extrapolated combined structure and function of processes within the physical environment from heterogeneous volumes within heterogeneous volumes, derived from a functional set of concepts, sharing the same ontology is necessarily dependent on witness encounter with other witness and/or with a common environment to both, must necessarily bear the condition that all witness can be ascribed to the existence of a commonality with nature, a oneness, identity as a necessity for witness and cognitive interpretation. Capture of combined elements of structure and function to the particulars of given setting is not considered to be a practical possibility, though temporal processes are postulated to entail, regardless of the potential existence of a common universal origin, the existence of an unwitnessible, hence parametrically incompatible for inclusion in whole description, common element from which the descent of energy has occurred, i.e. an inheritance by proximity in which all relations are enabled by a process of familiarity. Combined renditions of structure and function are theorized to be uncontainable parametrically in order to interpret unique structure and function with respect to the interval of time, the perspective dependent situation. For example element captured to, for example ranges given between 1 and 100 will necessarily entail for closure of the entire scene to a given geometry not only other elements with ranges in perpendicular arrangement in orders approaching endlessness, 
beyond the plane of description, but beyond witness for measurement; a vastly influential and stably experienced power, immutable as a shared condition of system state by all elements, as a fulcrum of the absolutely unwitnessible upon the witnessed present is suggested. Precluded in this interpretation are constant numerical/empirically measureable values that can be attributed conceptually as universal state variables. Such variables, i.e. a constant velocity of light, necessarily refer themselves to situations that are the consequence of likewise situations modulated by shared containing elements of both, are historically bound to propagated witnessible situations to produce a sense of simultaneity that appears to have potential for elaboration employing mathematics that gives representation resembling the action of a fulcrum. The former, a vast lever applied by unwitnessible elements is a matter of imagination, bears resemblance in form to the latter that is a matter of induction that ensues from witnessible phenomenon. Though a physical environmental sphere denoting familiarity of all elements in the theory of relativity is possible-i.e. the earth, its' inhabitants and forces acting on the environment- philosophically it does not render a viable comprehension of the relations of nature, men and nature that is suitable to maintain footing needed for survival, entails a surrender to universal status of an assumption concerning the nature of physical processes as they influence earthly conditions, as understanding is applied towards solution. Uniqueness of the elements of nature entails a force of self avoidance that is modulated by contrast; the self, not different from the self, possesses greatest familiarity, least difference and greatest compatibility, sharing of physical and conceptual elements, even globally, within encounters reflects shared mutual witness and a necessary existing familiarity that does not reflect philosophically all familiarity dependent witness possibility. For interpretation, description is limited to begin and end with the self, with the first perspective, either to entertain the possibility of suppression, denigration of the self and its' spaces as a consequence of the induction of universal standards based on witness that is necessarily restricted to its' temporal and physical location.

Employing mirrors the self cannot witness itself simultaneously in its' own reflection and a reflection of its' reflection but in very immediate proximity to both mirroring surfaces, within close range to surfaces; it is supposed that this is a universal consequence of evolution that brings about the forces that act to maintain an integrity of the entity. It is at the intersection of obstruction to self witness that life, volume, physical form is postulated to be birthed, to function as an obstruction to the death of an energy of creation in a process of avoidance of loss of identity, negative selection, rather than from positive selection oriented towards the direction of life, growth towards the open. It might be speculated that the branches of a tree grown from its' seed might never align in a parallel fashion: the connected branches of a growing tree, derived from a common physical spatial-temporal element, in opposition to a tree of evolution or discrete parallel aligned association of living entities, aligned in parallel arrangements entail circular, closed paths that preclude time and diversity within the depth and breadth of their branches and roots, and negate the prospect of growth and development; parallel physical lines of growth, postulated not to intersect anywhere in space, might not produce the connected branched structure of tree, the potential for the emergence of obstruction of death effected by threat of simultaneous multiple self witness at great distances logically could not occur. 
Descending from a common containing volume, the conceptual and the physical assume an independent relation to it. Though not all species are observed with the capacity for cognition, it is proposed that the concept has a universal existence and nature that is independent of the human capacity for thought; to possess a specific character that is a parametric property of (becoming closed) spaces that is transmitted temporally to a set of constantly closing heterogeneous spaces. It is in this sense that it is thought that the 'ether' postulated as an entity in the physical sciences as a the substrate for the propagation of light, can be substituted with the notion of 'the concept'. A world construed as a construct of need and symbolism and an atemporal process of mirroring is proposed as a sufficient universal conceptual outline suited to account for all explanation; it is composed of an interchangeable, between energy and matter, physical form, of memory as states of energy-matter that establish relative endurances of structure that is necessarily reflected from tangible factors related to familiarity that is present in different degrees and is possessed to all of the species of an environment, whether or not cognitively able, living, inert. Symbolism reflected from need to avoid loss of identity, death, that occurs from a loss of energy embodied to maintain it, might suffice to inclusively account for all energy metabolism that is inclusive and pertinent to the first perspective, to the set of all first perspectives. Simultaneity is redefined, not to refer to a temporal simultaneity of events but to a holism that simultaneously functions at all levels.

In this model, open space as volume is not taken to be absolute parametrically, but relative to the event of witness such that totals are construed to be a facet of total available witness and is not absolute; in the absence of witness a volume can be construed not to exist, the total volumes pertinent to the earth can be reduced in definition to those volumes that are witnessible to pertinently associating agents associated with the earth.

A volume of space can be attributed an identity as a synergy modulated by the interaction of individual volumes: a parallel can be made to the cultural trait conceived as conceptual volumes, either witnessed physical/natural volumes or cultural traits can be given a relative empirical nature that is a relation of relevant and necessessary witnessibility-e.g. sets of ratios of single traits to wholes and related to construed survival priorities. The dimensions of a 'road sign' can have a similar dimension in magnitude to the road itself, can be traveled upon and thought of as a road though maybe existing as only signs representing superfluous branches of effective processes that bear real and actual significance: 'road' or 'tree' as description has no implied dimensions though by deception from inductive reasoning conceptual branches of processes engaged in the emergence of structure can be given existence in other than a parallel arrangement with the path of processes attributed to the individual; it is only the physically connected organization of the branches of trees that necessarily endure non-parallel arrangements. The designation Hiway One or an angle of bend to a ray of light, the velocity of a light beam, has no numerical content that can belong to conceptual frames; the numbering system describes rather than undergoes, as a physical road or path, emergence. Parametric volume thus is not only dependant on measurement with (relative) standards but on the importance of particular volumes to survival, on their relation to witness as discrete points 
falsely included to conceptual and not physical (tree) structures, to other than volumes where distinct multiple self witness potential has resulted in birth of the location, the self, is localized to the physical self and obstructed in long projection to reside in limited physical areas; the self, the environmental niche, the earth can be viewed distinctly, each with varying ranges of potential simultaneous multiple self-witness, the whole range of familiarity based potential witness to events pertinent to the content of particular region may not necessarily be realized from encounters.

Though relative physical size attributed in this manner to material objects may escape logic, it is construed to be real and applicable as observation and measurement is ubiquitously contained to the facets of the physical and conceptual, to the acts of witness that occur meaning based on need in denominations associated with witnessibility; it entails time as an abstraction only that has empirical meaning as a relation that is relative to the experience and history of the path accumulated to the life time of engaged witnesses and empirical facts of their association.

This model in which a universal process of obstruction to events of multiple self-witness is postulated to produce a reduced range of witness, volume and defined shape of the entity appears suitable to accommodate all description:

1) The genetic material DNA, its' arrangement into chromosomes can be considered to be the effect of energy matter conversion resulting in "physical pieces of energized path"i.e. DNA. The existence of genes, the genetic code as a facet of their life time ratios to whole structures and is related to the volumes of entities and associated processes. The presence of uracil in RNA rather than DNA can be viewed as a natural evolution of an energy possessing physical divide between the contemporary self witnessing components of the cytoplasm and the long life time of inherited structure to the genetic material in the nucleus, as the simultaneous birth of a form to energy from physical obstruction to threat of the loss of energy bound to form. Biological identity is occurred from a path of energy approaching multiple perspectives capable of multiple self witness. A complex spinning machine is envisioned to be entailed that can be accounted for by processes of negative selection against loss of identity death, the closing of spaces.

2) In the physical sciences it might not be said that a measured value for the velocity of light can have coherent meaning if it is not referred to both the measurer, i.e. mankind, the human species, and the light measured; the witness pair man-light is not the same as the witness pair dog-light, or light-mankind, light-dog, Albert Einstein (1986) and reflected light or Max Weber (2005) (who was not a scientist) and reflected light. Actual problems of civilization, other than directly approached, might be viewed to be referred to in a symbolism of living/acting out, of preoccupation with the elaboration of physical laws that have no objective formulations to include the individual, accommodate evolution other than as a thermodynamically illogical, mysterious emergence from inert materials. Relevant to interpretation and more important than searches for constants to define universally nature, though it is unlikely that birthing 
energies of environmental spaces that contain the earth and its' inhabitants are witnessible, might be ratios of lifetimes, energies of observed light to those witnessible, measurable of the earth, species/individual, the pursuit of a relationship to physical particulars of potential unknown problems related to the earths' and individuals' place in the universe and its' processes

3) Cultural evolution, cultural concepts that have come about from sensory experience of the witnessible physical environment (Clifford, 1922, Fortes and Evans-Pritchard, 1940, Bourdieu, 1977, Sachs, 1982, Strathern, 1990, Marcus and Fisher, 1999, Geertz, 1963, Harris, 1979) can be related similarly as paths arisen from processes modulated by familiarity as the primary criteria for evolved behavior; cultural traits as a range of, scaled based on prominence, ratios of individual traits to established total culture specific compositional differences. .

4) Economic systems and their aspects can be described with respect to witness, witnessibility as the basis of criteria for estimations of potential value as an emergence from the possibility of conversion of accruing conceptual capital assets to material assets. A universal form simultaneously the agent of a natural economic balance and the shape of a elementary natural food source, the egg, can be derived strictly from criteria of physical existence; is dually both a geometrical form possessed to energy and a physical shape of biological spaces, is postulated to be a fundamental shape of all spaces and can be elaborated from notions of constraints to spaces as necessity for their existence.

If the total of external physical volume pertinent to activities of mankind changes with population statistics, size, geographical distribution, it is not possible to speculate that space exists as a fixed entity, but is dependent on the variables of witness. If the volume of space is effectively variable with respect to time, age and state of a witnessing population, all existing subset volumes may be considered to be parametrically variable for effective description; historical interpretation may be plausible only when ratios relating the observer to the observed entail the same library of concepts that has remained pertinent over generations, i.e. if the same family and environment is relatively unchanged with time; homo sapiens and the earth, maintained as topics over the interval of comparison can be validly assumed to possess a qualitative relatedness in categories employed to establish correspondences for comparisons.

In analogy to the geometrical renditions of Euclid, in which the parallel situation of straight lines cannot be contained to mathematical proof never to intersect (Euclid's Parallel Postulate) it might be speculated that a valid continuity of identity of earth-mankind or earth-biosphere might exist if it is assumed without proof that identity of all entities and concepts involved in witness relations, both past and present, descend from a commonly existing intersection to a common and similarly contiguous containing element, i.e.-identity continues from the indivisible point(s) of witness, environmentally modulated, propagated witness relations as a divisible line that extends towards the parameter bearing temporal intersection, situation, and is strictly conceptual/philosophical in nature to define permissive intercourses and their plausible 
continuances, to delineate compatibilities from a range of emerged/emerging familiarities for all intercourses from which the generation of new combinations and diversities emerge. It might be inferred that Euclid and modern theorists refer simultaneously to the same earth. Necessarily intersecting rather than parallel arrangements of paths and physical contours are held to be the only existing case in nature. Euclid's adherence to intersecting lines in his geometrical renditions, resulting in the inability to prove the Parallel Postulate, might be taken as testimony to philosophy based on the existence of conceptual parallels and the non-existence of actual parallel lines in nature. Interpretable meaning, dependent on the conceptual parallel, given to ancient written creations might be held as evidence of this possibility. Within associations drawn from hand written communications, true parallel lines, indicating nonexistent/false correlations, are postulated never to exist if a relationship, past to present, seem to be established, though witness involving the past is not available. In this situation, the (evolved) hand is left grasping, attached to a temporal motion of the indivisible point, as if to the nonparallel branches of a tree, as it elaborates a punctuated infinity, as the only evidence of existence, all else failing as a fact of the simultaneous possession of, incommensurate with one another, measurability and non witnessibility It is thus considered profane to take as evidence in the interpretation of existence that which exceeds the perspective of the individual as easily as it might be construed to be profane to base truth on invention or the employment of additions assembled from limited witness to define wholes; all that can be relied upon for the grasp in valid comprehension is the absolute existence of the conceptual parallel and the un-provable absence of the physical parallel. Space itself, strictly possessing non straight intersecting physical lines, cannot but be composed of force bearing unwitnessible parallels of a conceptual nature existing, unwitnessibly connected in tandem/parallel with strictly physically branched structures.

It might be speculated that the earth or solar system, possessing a finite life time is the result of the physical uniting of branches of processes, the consequence of a fitting together of separately evolved structures upon the invasion of spaces possessing a familiarity with one another that is the consequence of the existence of a shared containing space from which they had emerged and migrated into proximity. The continents are thought to have rearranged physically over longtime intervals (Becker, 2010), certain simple linear migrations occur to the earths' core (Alboussière et.al 2010) (that might in addition be speculated to bear an association with forces responsible for false theoretical constructions of nature). The human brain might be speculated to have arisen similarly, as the consequence of motions, diffusion and uniting by proximity of physical elements or branches of distinct and physically separate, hence seen or experienced paradoxically parallel, unconnected, to one another, resulting in a species with depleted olfaction ability and a second olfaction, cognition that is constructed of parallel associations with connections that are permanently obstructed and unwitnessible, a happenstantial process of pursuit towards the available open and consequence of necessarily occurred physical restriction to distal simultaneous multiple self reflection witness events. If the nature of the concept, thought or cognition is construed as a process similar to the physical process of diffusion, in light of an appearing lack of evidence to establish a physical evolutionary path of H. Sapiens, especially with respect to connections for the emerged rearrangement of the head and cranial vault, reduced prominence of olfactory abilities in $\mathrm{H}$. Sapiens in comparison with other species, certain facts might emerge with respect to the evolution of cognition. When the nature of evidences from the fossil record are considered, all aspects, without exception, are dependent on the 
possibility of diffusion of elements as the primary process in the production of unearthed records; this includes evidences involving the nature of formation of fossils, the conditions required for their formation, locations of their discovery that are related to land upheavals as the source of weather related changes in ecologies, the means of extrapolating weather data from studies of distant ocean sedimentations, speculation concerning physical migration of land areas, theoretical controversy involving Out of Africa and Multi-regional routes (Johanson, 2001). Despite the dependence of evidence on the existence of conditions suited for their preservation, human origins may be logically confined to a happenstance of open spaces and strictly to the type of places where they are currently found. It is perhaps due to a similarity of the nature of cognition and olfaction, that researchers are logically obstructed from unearthing evidences to support theory based on direct physical chains of cause and effect; cognition abilities in modern man, endowed from the very occurrences under pursuit, may be blind to elaborate directly from physical evidence the details of their origin, at best only profuse physical evidence may be found for reflective postulation. In less rational or less scientific, though not profane description, as no tangible connection is made in numerical order from the invented to the conceptual, mankind might be speculated to have diffused into existence in a descent from the physically existing location tree (i.e. point of beginning). It is interesting that the evolutionary species tree discussed above, lacking totally cognitive ability, depends for reproduction, on random physical dispersement, rather than physical contact, for the intercourse of seeds.

In Euclids' writing the indivisible point, envisioned to the end of a divisible line, has no continuity with other points, it exists alone and is not considered to be a contiguous part of something else, e.g. a line that is divisible. (Morrow, 1970). It is from the indivisible point, the concept of physical connection (i.e. the tree structure) and the concept of diffusion (i.e. the concept itself) that parallels can be constructed between the frustrations of civilization, the diffuse uncontainable power possessed to dialogue in the processes of planning and change and efforts to understand nature and the world in a rational manner. Necessarily obstructed by the self, in the act of perceiving both the tree and space as open, space with greater physical room, in response to intrusion to their spaces, men simultaneously, symbolically push themselves from 'the tree', a symbol of cause and effect, the scientific, rational place, point from where they are supposed to have descended, and causing witnessible physical damage to nature, pursue injuring concepts, themselves indestructible, with aggression that has a door stop necessarily beyond witness, 'the concept', itself that is obstructed by the incomplete concept of self as it has become structured within relations peculiar to the culture of the individual. In this light, the fervent, though unnecessary to an understanding of present conditions of civilization, search for human origins becomes more understandable as it has become an object with to fill an incompleteness that threatens survival, existence, as a prosthetic for the grasping hand to find a source of his conceptual abilities in order to quell destructive behaviors and energy that are currently focused upon the annihilation of injuring and enduring concepts, the consequence of the experience of encounter; to correct history with acts of annihilation. In this sense the theory of relativity, referring vastness, distances and mass together, to a fulcrum constructed of mathematical relations, assumes essentially a vastness and time capturing mythological character that presents a potentially volatile social situation. 
Volume is here recreated to refer to emerged synergistic parameters of unique common loci that possess conceptual identity in however many relations of witness that may refer to them. The bumble bee, necessary to all humans is reported close to becoming extinct (Latsch, 2007). In this discussion the volume of the bumble bee is not a grossly estimated 2 or so cc (cubic centimeters) that can be measured physically, but a $2 \mathrm{cc}$ volume that is distributable throughout the entire human race ( the survival of the bee affects the survival of every human) as a single volume that is adjusted by a ratio that reflects it's priority in a set containing all enumeratable volumes that are likewise pertinent to the members of a culture that refer to them. For instance, if the number of volumes pertinent to all men is inclusively 23,256 and the bumble is rank \# 2 in order of actual prominence to survival then its' effective volume would be adjusted, regardless of actual physical measurements, to $23,255 / 23,256 \mathrm{X} 2 \mathrm{cc}=1.999914 \mathrm{cc}$. If the extinction of the bee is the result of an act of genetic tampering, involving the employment of an actual measured volume of $2 \mathrm{cc}$, the hands of the researcher upon the genetics of the bee reduced the real volume of space occupied by the bee to the effective difference of $1.999914 \mathrm{cc}-2.0 \mathrm{cc}=-.000086 \mathrm{cc}$ of lost environmental space per existing bee for each bee lost. If a randomly estimated100 trillion bees become extinct :

$0.000086 \times 10^{\wedge} 14=8.6 \times 10^{\wedge} 6 \mathrm{~m}^{\wedge} 3$ of volume becomes absent from the world If the volume of a man is $1.6 \mathrm{~m}^{\wedge} 3(\sim 2 \mathrm{X} 2 \mathrm{X} .4) \mathrm{m}^{\wedge} 3$ and 1 trillion men exist $=1.6 \times 10^{\wedge} 12 \mathrm{~m}^{\wedge} 3$ of total volume of man each mans effective volume will be reduced to (an unwitnessible, unmeasureable) $\left(1.6 \times 10^{\wedge} 12-8.6 \times 10^{\wedge} 6\right) / 10^{\wedge} 12=1.5999914 \mathrm{~m}^{\wedge} 3$ $=-00.0005375 \%$ reduction in the volume of men

It might be postulated that the loss of the bumble bee is occurred from an autoimmunity problem of the same nature that is witnessed to be lived out in the actions of a consented asceticism supported by a blinding repressing obstruction to the conceptual natural self in which capitalistic enterprises result in natural abuses that in turn had prompted the research in molecular genetics that enabled the tampering with the genetics of the bumble bee in order to preserve a farmed species for cultivation that is lesser in priority for survival. This cycle of the willful transmission of the form of an occurred impulse, profane to rest its' case strictly on invention by means of induced points of destructibility based on connections made by a physical geometry adapted to the ideal free floating figure, can be interpreted to reflect an evolving cognitive blindness to the possible existence and nature of possible (unwitnessed, unnoticed) events as the instigators of self defeating behavior. It is not logically excludable, if logically arguable, that historical paths of behavior might be traceable to other than diffuse phenomenon, ordinary nature, but protracted externally, occurred physical impulse from unordinary nature: false attribution of physical elements to conceptual categories in renditions of nature arises to produce theory that bears false assumption and connection of observed facts to concept.

Scientifically elaborated paths of processes may be but the consequence of the paths of other processes that more pertinently bear on conditions and have a different etiology than is rendered from study of its' branches. In this interpretation it is interesting to note that roots of self defeating behavior, i.e. physical impulse from a pathological nature, possessing no active voice in discourses, not emerging in theoretical considerations, can result in the presence of a tangible 
obstruction to the expression and realization of appropriate social spaces with which to work resolutions, can also evolve to be self defining, existing of its; own as it is confused with actual nature. An existing, self or majority imposed vicious cycle arrived from frustration to contain observation and experience to theory, then motivates group efforts, frustrated for explanation, to unearth a paradox of the material and philosophical, of mind and matter that is evidenced with a flurry of scientific, medical investigations into the brain, consciousness, yet with the guiding eye focused upon the same means and tools employed in a motion along a finite historical path that is ridden with confusion involving artifact and nature. A potentially preexisting autoimmune deficiency, an invasion of natural, personal spaces, from which evolved incentive and means for the elaborated genetics of the crop pollinated by the bumble bee became the subject of manipulation, functions to obstruct efforts towards resolution. Western methods of reduction and repetitive division, to expose conceptual divides that in older societies are assimilated apriorily in symbolism, granted to natural order, in either case allude to the application of impulse to control as resolution to inflicted natural suffering. Ethics, that have come to focus on a lack of regards to the rights of others in the wake of failed efforts towards a natural ethic, are unsturdy, insufficient to effect changes in behavior. Acculturation process in which contrast with respect to the application of impulse to effect natural change does not exist, economic conditions favor the mining of nature, companion an illusion, myth of confusion of open space with the branching structure of the tree, a modern science with its' eye on a perceived infinite open rather than the finite space occupying ill-defined individual, to possess the materials needed to repair branches broken somewhere upon the ascent of civilization.

These kind of formulations in physical models that are mechanically ruled from existence, men made to be machines in the same manner that a comprehensive understanding of nature can be conjectured for learning from its' isolated parts, at the same time to include and exclude in temporal order, self and space obstructing, inductive reasoning, as simply as volume of spaces can be over estimated and sheared from existence as in the given example of the bumble bee, potentially result in cultures so modified by intrusion, that actual problems become buried beneath a predefined and socially propagated mechanical breath of observation in a mode of operation involving the enormous consumption of resources at the ubiquitous border of conflict at the intersection of the rational and the spiritual/religious. Etiologies involving different cultural symbolisms based either on a prominence of discovered structure verses function, the energy invested towards inherited structure verses energy invested metabolically to maintain structure might be construed to refer to a common problem; it is possible to speculate that an auto immune deficiency related to the energy involved in metabolism involving the ingestion of self, cannibalism exists is constantly emerging in a temporal direction to be more prominently expressed in representations of nature in newer societies. A potential gene spread throughout mankind, associated with cannibalism, has been reported (Roach,2003).

A situation for a universe that pretends the universe to be only what is witnessed or experienced, witnessed history as a whole that possesses location based anomalies that are mirrored externally in reflection of it seems to have been excluded. In alternative, with the analytical eye focused strictly upon proving evidence at each step, attention placed almost strictly upon the physical, an exchange of order of arrived concept and physical evidence is occurring in which the eye necessarily needs to focus on the straight line as an indication of the path of maximal 
open, greatest lifetime, has instead taken a long and winded route of a living out in that is in compliance with its suffered problems, to slowly realize them to actuality for discovery only at very late stages. It is interesting that it has recently been observed, surprisingly, that chromatin carrying the genetic material in cells contracts rather than expand on pulling (Fulcronis, 2006); matter internal to the cell, whose properties are sought in explanation for functioning of the whole, might not be expected to be organized itself in a manner that appears to contradict established laws of thermodynamics when applied to evolution, to possess what appears as an animos that bears resemblance to the behavior of the individual entity. .

A philosophy to parallel the human situation, 'the circumstance' must be non spectacular not to engage the silent spectator but to account for him. Though it maybe that matter, volume, space, are always in the process of being created in the niche and perspective of the first person, regardless of the identity given the agent(s) responsible (which non-the-less can only be the same as what is created as it is all that is present ), the verbs 'created', or 'discovered' employed by either the Post Modernists, Logical Positivists, or the rational scientist, bear innately an association of the meaning of truth with action, necessarily entail the application of power/force to the elements in a philosophically incompletely addressed path; argument over intelligent design theories between the creationists and the scientist who bears intelligent designs that are blind to the potential existence of valid conceptual spaces that include it's own symbolisms, are likely to produce only the domination of a philosophy purporting the employment of a logically complicated and empirically supported view of a world that is physical impulse dictated endlessly from the necessary beginning of a discrete point of thought to effect a linear crossing over, exchange of the physical and conceptual as lending seeds of understanding. The present situation of contest of rational science with religion, places both with the responsibility of, natural damage occurring, pursuit of injuring concepts, evidence both in social aggression involving ethnicity and religion as well in purported solutions involving scientific methodology. Battle over the existence of god ensues, a spiritual lifting from within verses frustration in more complex argument evolved from an inability to overcome gravity, to claim aggressively from a position of practicality, within the light of the professed and philosophically predicted absence of witnessibility of a gravitation-less control for study. The struggle to argue intelligent design verses evolution is difficult to conceive without the involvement of twisted, falsely based unwitnessible criteria in arguments. If the study of self-unkind man is structured from a perspective in which the study of anthropology has the property of self belonging (Kirsh, 2009, 2010), if the act of studying mankind belongs to a set described as mankind, then contradictorily, so must the activities of self-unkind mankind to a set "self-unkind mankind" that can be no different in correspondence to the self belonging set 'nature' as the inclusive set of unique gravity possessing volumes.

It is interesting to note that description involving problems intersects strongly with ontology in modern genetics. Terms such as deletion, (unequal) crossing over, codons and the punctuation in the reading of information by internal entities such as replication, transcription and translations processes are easily applied to descriptions involving the abbreviation, obstruction of the infinite line to produce form. It might not be surprising to find examples of the discussed pathology that is conjectured to be mirrored from tangible localities of spaces that influence the earths environment within the genetic material. Inversions as well as deletions, duplications, are commonly known mechanism of evolution, inversions are associated with vision, color 
blindness and other pathologies involving vision (Carvalho et. Al. 2010, Seoighe et al,2000) . It cannot be logically argued that phenomenon observed in the laboratory are restricted to the conditions of experiment. The converse might be intuitively argued that all such phenomenon have occurred in nature as a prerequisite to the course of topics and experimental serendipity involved in their discovery, as testimony to the inquisitive mind that is guided along a path of historical behavior that is practically centered on the physical, unawarely influenced by that which is unwitnessed, at the meandering edge of full comprehension of meaning, instinctually taking notice of pertinent clues to toy with them as if they were parts of an inexhaustible open, planning a course of rationally contained pursuit from which unhappily surfaces spectacle of interpretation involving cause and effect after exploits, a failure of the science of preventive medicine. As a hypothetical example, if a laboratory worker is able to cause a retraction of chromatin upon pulling on it, it might be more advisable at start to pursue evidence for the natural occurrence of exogenous physical agents to have penetrated the nucleus to cause pathologies, than a meticulous investigation into the nature of the genetic material. In the case of cloning research, other than to toy and construct, to seek evidence of unnatural, pathological occurrences that might potentially have ensued from external physical problems of the environment.

Entailed within all these described gears is the need for a revised scientific approach that is silent, visual, in the wake of a vast blindness that currently occupies steering mechanisms. A single action of replacing the conceptually heterogeneous entity of time, with the unwitnessible entity the mirror as the generator of form of is presupposed, less in a search for the dark and silent, only the dark results.

If it is possible to construct descriptive properties of path from path bearing witness testimony, it should necessarily include an accounting of a necessarily existing unwitnessible, hence, unknowable containing element as the influential agent of criteria of description of both all of the contained physical path and associated ensued path of concepts. A suitable representation is proposed that is accomplished from geometrical representations that elaborate path in terms of a radius that is construed from a moving circular radius composed of parameters of the two kinds of important energies known, from the slow observable motions of matter and fast, either observable only with respect to a consequence effected on the former, or unobservable as the path possessing agent responsible for the becoming contours of the physical environment from which conceptual structure is evolved. In a further arrived strictly from philosophical considerations, the unwitnessible path of the concept can be given existence as a displacement, a difference state, arrived from physical proximity of unwitnessible becoming states of energymatter conversion. Figure 1 illustrates an oval as a generating template for the construction of physical volume from fast and slow velocities of motion; becoming matter is defined by a change in the velocity of light emitted from a moving plane along a line of reference. Figure 2 shows an egg shape that is generated from the oval template in Figure 1 employing a combined expression for energy from Einstein's special theory of relativity and Newton's laws' of motion to calculate a form generating radius from linear motion of the radius of spheres constructed solely of energy considerations at fixed rather than moving perspective.

$$
\begin{gathered}
\mathrm{E} / \mathrm{m}=\mathrm{v}^{\wedge} 2 / 2+\mathrm{c}^{\wedge} 2 \quad \begin{array}{c}
\mathrm{c}= \\
= \\
\\
\text { of energy to mass }
\end{array}
\end{gathered}
$$


Volume is related in symbolism as (slow) $\mathrm{X}$ (fast) $\mathrm{X}($ fast $)=(\Delta \mathrm{c} \Delta \mathrm{v} \Delta \mathrm{v}) /$ time $^{\wedge} 3$

The Concept is related in symbolism as $\Delta(\mathrm{c}-\Delta \mathrm{c})$ (unmeasureable and unwitnessible) to reflect a difference of system states with time.

The witness pair is construed to necessarily be always present within the relationship of emission and reflection, the egg shape as the limiting form results from the conversion of the energy of emission to mass The resultant egg form illustrates the naturally existing situation in which the tangible world is given motion to existence and requires that the orders of magnitude of change are interpreted to distance; time, as a parameter is deleted and assumed to be a perspective dependent state variable determined by witness pair location dependent factors associated with events, is replaced by a mirror that reflects need and acts between associating witness points. In the graphing situation periods of pi $+1 * 10^{\wedge} 17$ revolutions are required before visual witness of the egg can be realized. The required presence of pi is interpreted to indicate closure of the whole surface that has no geometrical center (other than the line $(\Delta \mathrm{v}+2 * \Delta \mathrm{c})$ and a relation of coordinates of sine and cosine; a closed surface is not represented but from a coincidence of parameters of plot path, sign and periods of revolution. The large numbers of periods of revolution are construed to represent a relatively simple, in light of the complexities that are entailed, but vast temporal period required for the accomplishment of physical nature. In a test of graphing parameters the egg shape and general overall appearance is variable and dependent on input parameters (not shown). The golden number or similar ratios representable with numerical square roots and trigonometric values combined, result with similar but different structures that can approach to be indistinguishable from a true sphere but lack a mathematical center point. The egg, constructed of witnessible slow motion and unwitnessible fast energy propagation is presented as an elemental shape for space, suggesting that the shape of the ordinary egg, arrived from the maximally open straight line (i.e. $\Delta v+2 \Delta c$ ) rather than a curve or circle represents a structure that has the maximum lifetime. The egg shape presented is employed to function conceptually as a bridge between conceptual, philosophical and material reality, as a concept that can be related to physical existence. In an extended analogy to the circle of Euclid, a philosophy of motion to Euclids' geometrical rendition of connections and attachments of lines and planes. In parallel to the translation by Proclus describing Euclid to construe the circle to be composed of indivisible points rather than a divisible line, the egg is viewed to be the limit of the sphere (circle), i.e. the sphere and hence the egg is the circle possessed with breadth and depth of motion.:

"One might think that, although both the straight line and the circle are simple lines, the straight line is simpler. For it contains not even any dissimilarity in thought, whereas concavity and convexity in the circle indicate difference; and the straight line does not suggest the circle, whereas the circular line does bring to mind the idea of the straight line, if not through its' mode of generation, at least by its' relation to a center. What then if someone should say that the circle needs the straight line for its' existence? For if one end of a finite line remains stationary and the other moves, it will describe a circle whose center is the stationary extremity of the straight line. Should we not reply that what describes the circle is not the line, but the point that moves around the stationery point....It appears that the circular line belongs with the limit and has the relation to other lines that the limit has to all things...the circle alone is limited and makes a figure, whereas the straight line belongs with the Unlimited and can be projected indefinitely without end " (Morrow 1970b). 
The 3-D egg is viewed to be indivisible as the circle is to the center point and motion. It is motion, beginning from the indivisible point of motion contained to a thought, in a movement towards the elaboration of witnessible form that is the theme.

At the point of departure from the philosophical towards the physical, efforts towards the accomplishment of a concept and definition of time, not only intuitively occupying, necessarily occupying space and time in a description of space and time are here envisioned to be obstructed from the first perspective at all perspectives, by the self that is composed, in its' elements, of the same, molds activities involved in the occupation of physical space, and are in correct order, philosophical form precedes physical form. Hopefully, an interval of creation, the interval of time in its' simplest form is envisioned within a process involving the making of lines on drawing paper.

The rectangular table I am writing at is filled with boxes, within boxes, spaces that ultimately do not have the form of the box that is intentionally molded by impulse of hand or machine into the construction of the table and bear only in common the possession of a geometrical form that has both a physical and abstracted nature. A truth exists of the interval of the writing of lines or words upon paper that rests on the table; for truth, the action of writing lines, the path created in the interval of writing cannot deviate far not to match those of the creation of life paths and reflections from which our learning and concepts are evolved. If space is assumed to be representable by a shape, as in the geometry and divisions attributed to the square table, though universally more round than the square, persistence with square figures in representation and interpretation of situations can make, a hard to see from historical actualities, spectacle of the propensity of the human hand to draw rough edges to continuous curves or visa-versa, a false spectacle of testimony that is enabled correspondingly to unknowingly trim fact from surfaces as easily as a criminal is born to seek to cut corners within a frustrated situation.

... And thus we must think of the plane as projected and lying before our eyes and the understanding as writing everything upon it, the imagination becoming something like a plane mirror to which the ideas of the understanding send down impressions of themselves. (Morrow 1970c).

It is thus that philosophical struggles with ideas of structure and function, obstructed in range by the always present self, as in methods to elaborate cultural evolution, can be envisioned to never fall together for witness in a combined fashion to account for specifics in the passage of time; the time elapsed to the individual in the rendition of form, is a creation, synthesis, of the new that has, as unacomplishable boundaries for creative cognitive description of situations, an absolute shape of space as a limit that includes the self within the irregular dynamic intercoursing, containing and contained spaces of the individual and the physical elements of the environment; faithfulness towards philosophical truth cannot begin from the tangibly witnessed physical space with the employment of lines of cause and effect to render explanation; if a monism exists to the elements of nature it might be suspected to be hidden as a shape, it should bear in form content inclusively referring to the witnessible, the unwitnessible, and the unwitnessed. In this respect a strong relation of observation to the unwitnessed and 
unwitnessible that is characteristics of both diffusion processes and cognitive reflection as it is proposed to have evolved from and necessarily reflects diffusion processes, is demonstrated. The actual plot path of the egg surface from the ordered equation presented, as might be expected in valid interpretation is very irregular to the line of sight. The addition of a philosophy of motion in interpretation occurs in a manner in which the empirical is held as testimony to the philosophical in an analogy that does not strictly or necessarily align with indiscriminately induced empirically centered, self obstructed points that can produce self defining artifactual productions from the application of empirically centered willed impulse, but with independently found philosophical form; eggs in nature are neither free floating forms nor necessarily physically attached to their generators, but are bound in their creation, in the same manner as Ancient philosophers and mathematicians confined applications to witnessible intersections, to architecture, military etc., to the elements of the path of circumstance.

Nature is inferred to behave strictly in a living out manner and to follow a simple logic to effect organization that is construed as the propagation, necessarily both of a form bound to energy and energy bound to a form, as the propagation of force caused by an obstruction annihilation by the self, is able in the presence of pathology that is enacted, from the localization of self witness as criteria for the existence of the entity, to suffer premature death. Existing form is not so readily visible within temporally constructed chains of cause and effect from which the laws of the energy of processes are deduced.

Figure 1 An oval template for the generation of the egg from a simple geometry of motion of fast and slow radiation The egg (Figure 2) is evolved from a linear motion (v) from which light (c) is projected simultaneously in the $\mathrm{x}-\mathrm{y}$ and $\mathrm{x}-\mathrm{z}$ planes.

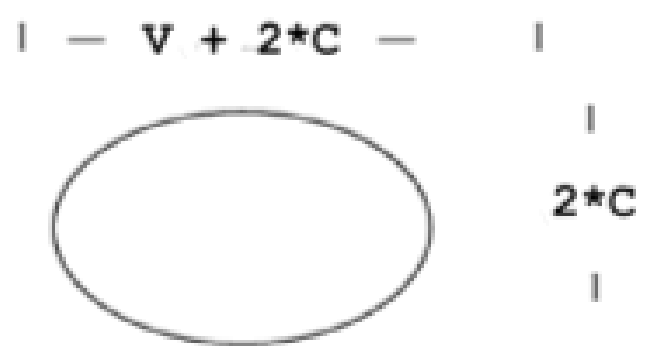


Figure 2 Three Dimensional Plot of the Shape of space is Egg Shaped.

$\mathrm{E} / \mathrm{m}=\Delta$ Slow Velocity^2/2 $+\Delta$ Light Velocity^2 (C (Speed of light) is applied as a

Variable)

$$
\begin{aligned}
& \mathrm{R}(\mathrm{a}) \text { Sin } @=\Delta \text { Slow Velocity } \\
& \mathrm{R}(\mathrm{a}) \operatorname{Cos} @=\Delta \text { Velocity of light }
\end{aligned}
$$

$\operatorname{Radius} / \operatorname{Radius}(\mathrm{a})=\left[[(\sin \text { theta })+2(\operatorname{Cos} \text { theta })]^{\wedge} 2+(2 \operatorname{Cos} \mathrm{phi})^{\wedge} 2\right]^{\wedge} 1 / 2$

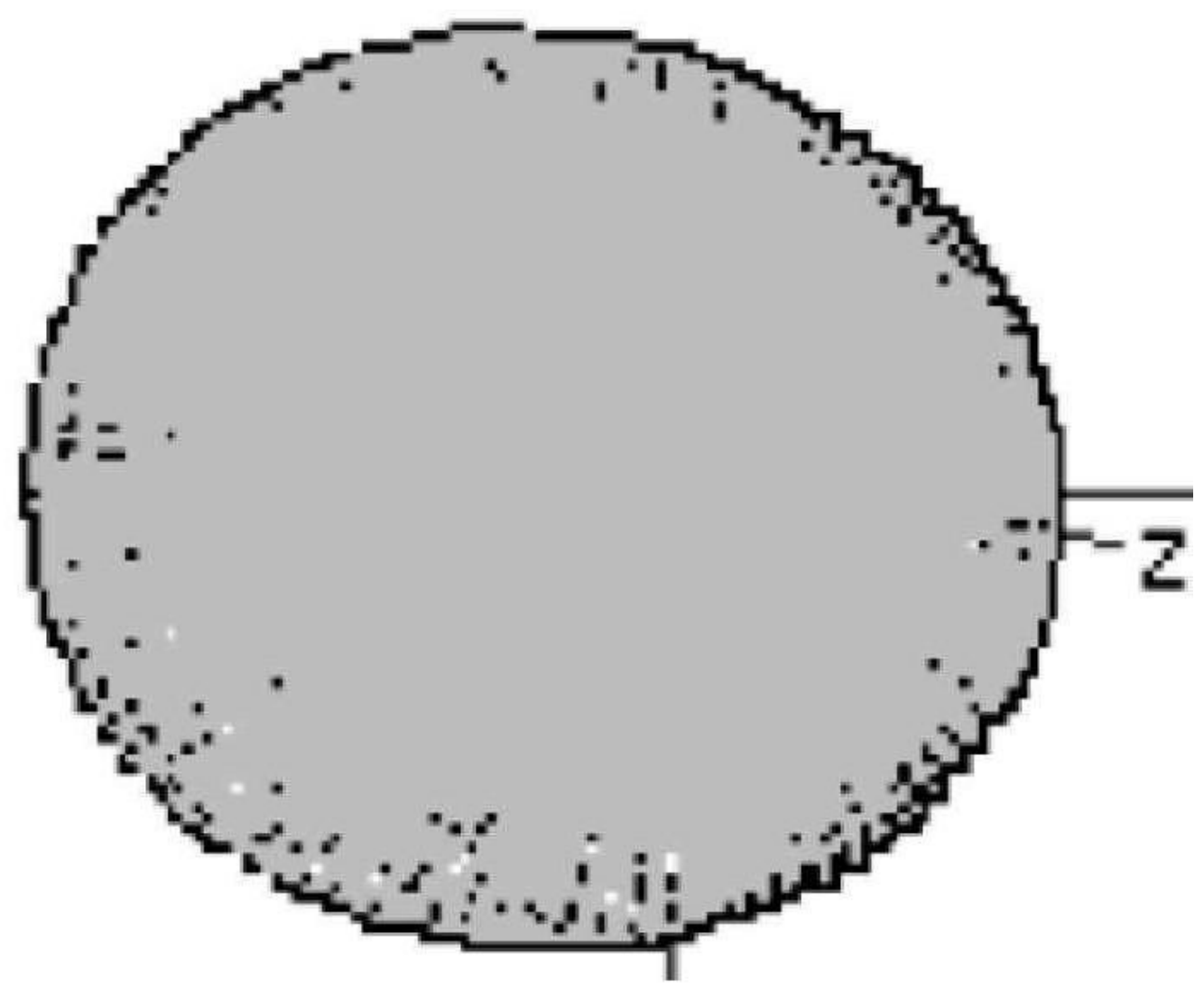

Nature, viewed as the intercourse, invasion of one by the other of independent, varying and diverse, self referring evolving systems of structure and function to produce more of the same, produces the historical intercourse, the 'situation' that bears a common ontology of itself, is proposed to be a reductio-absurdum fact of the obstruction of death by life.

...for the One is the measure of all things. So also in geometry the solid is bounded by the surface, the surface in turn by the line, and the line by the point, for the point is the limit of them all. In the realm of immaterial forms and partless ideas the line, being uniform in its'forthgoing, 
bounds and contains the varied activity of the surface and immediately unifies its' boundlessness, while in the realm of their [sensible] likenesses, the limiting factor belongs to the very thing that is limited and in this way furnishes it with boundary. (Morrow 1970d).

As testimony to deception, confusion and coincidence involving physical form verses conceptual form, it may be that as Karl Marx (Tucker, 1978) related that men are unhappy as wage earners in a self defining situation that is secondary in relationship to a defining nature, it seems more than seeking its' resources, seek to capture it to conquer it as if it had some mysterious hold over them. Possessing a synergy of the conceptual and physical, nature strikes life experience with two thorns when the conceptual and physical become confused, i.e. when the testable paths of cause and effect come to define nature as other than an entity of contained and containing elements seeking to avoid closure as the means of existence of the open. Experience renders to perception the possibility of non existent stillness and subsequent struggle to contain to rational logic the natural possession of an apparent animos to all elements that bear likeness to the self. It is important to note that cosmological views in which birth precedes death lend alternate perceptions and renditions of nature, natural history and human history, human behavior, than the reverse concept of death preceding birth. Most scientific theorizations struggle conceptually, continually, from a perspective involving the lifetime of the individual as it proceeds from birth towards death; this is especially prominent in theorizations of a big bang universal origin and theories of evolution in which accounting is attempted with respect to thermodynamics, explanation of the evolution of life as an assembly from inert matter. It is of interest that visual representation is accomplished by the brain from the input of metabolic energy to a negative image to produce a positive image. All of nature at any perspective maybe conjectured to work this way, is possessed with a divide of inside and out, the received, the emitted and reflected, the self as an obstruction,. Conceptualization of a universe that had arisen from failed neutralization of energy is not reflexively accomplishable from rational applications to witness experience. It is the accumulation of path to events, as the event itself, that is important (Kirsh, 2010).

"Thus the divine Plato said that geometry is the study of planes and contrasted it with stereometry as if he thought surface and plane were the same thing. Likewise also the inspired Aristotle. But Euclid and his successors make the surface the genus and the plane a species of it, as the straight line is a species of line." .... "For the straight line, he says is equal to the interval that lies between its' two points and the plane likewise...." (Morrow, 1970e). 
Figure 3 Symbolism of the Concept as a Mirror

The figure ' 8 ' is meant to represent a mobius strip, a continuous surface in which inside and outside are inverted and is meant as a partial representation occurred during the progression of path, the small darkened line along the figure is meant to represent arc length as it is involved in the priority ordering of concepts, an inverse relationship with respect to arc length as it bears on conceptual priority. 'The concept' as a difference state is proposed to reflect dynamic differences in the energy metabolism of spaces, processes.

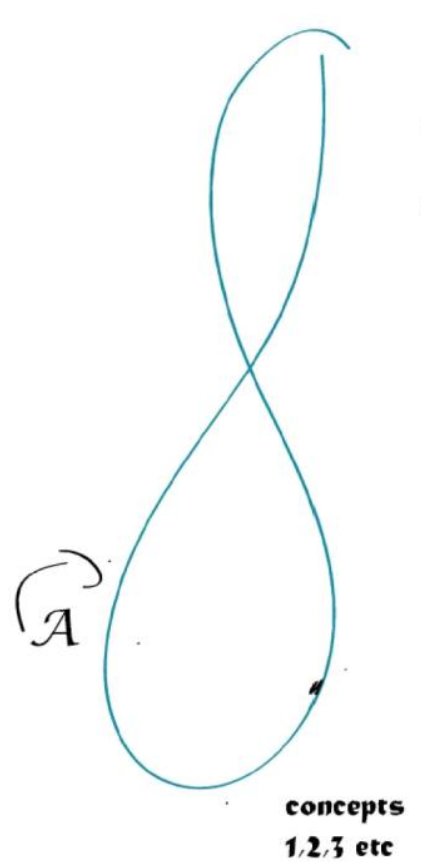

Toncept fias the shiape of thie mofius strip
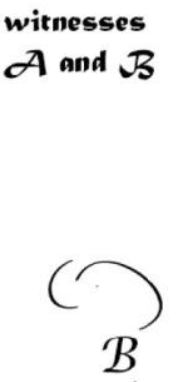

A prominent resemblance exists between the presented scheme and described molecular biological mechanisms for processes involved in DNA, RNA and protein metabolism. In contemporary theory, a rolling interaction along a linear surface, DNA or RNA, by egg shaped complexes in the cells interior, are observed in processes of replication, transcription, and translation to render new structure, to either copy DNA, transcribe RNA or translate protein. A model for the cell and its' machinery, as an arrived circumstance by proximal associations involving temporally modulated inheritance, is considered.

A tempo of events in the micro scale, where it can be assumed that the spaces around entities are experienced as open until death, is given as explanation for structure and function in processes that perhaps can serve as a general example of all processes, but with fewer agents and confined to reduced temporal/physical spaces. Within the relatively limited size of cells, oscillations and fluctuations occurring normally with vast diversity are correspondingly reduced from descriptive diversities observed in ordinary experience as is evidenced to the existence of a four letter genetic code that reads three letters at a time, or to a difference between an RNA possessing cytoplasm and a DNA possessing nucleus with respect to the presence or absence of uracil in genetic material. It is possible to envision a birth of life that is arrived from a necessary breaking of velocities of energy to avoid simultaneous multiple self witness, to birth matter that 
acts as a physical obstruction to the annihilation of energy from the accomplishment of a complete path. In analogy, simultaneous witness of the self to its' reflection and to itself within a reflection of self, as mentioned above, is possible in ordinary experience to occur only within a very limited range of dimensions in relation to dimension of the self. The self can be simultaneously observed in its' reflection in a mirror and in a second mirror reflecting the first only when both mirrors are held in close range. In this scheme, pre-existing conceptual form and demands placed on witnessibility and its' obstruction are the causative agents in the emergence of energy possessed to material form

In an imaginary vessel it is possible to envision the evolution of a molecular zipper from temporal processes that are on the order of factions of light velocities. During a process happening in a miniscule temporal range, and proposed to occur along a vast mirror represented as an inversion of inside and out, such as a mobias strip that has a twist in its' surface, in an a process of rapidly occurring change, mirroring actions across the surface, it might be proposed that obstruction of neutralized-fated energies pending disaster results in the emergence of physical species with temporal dependent varying degrees of relative twist in which points of emergence with respect to time of emergence determine the degree of twist, amount of energy with respect to its' absence in strictly flat surfaces, of emerged species and their fitting together. The mirror symmetry and twist to the uracil molecule serves as a primary example. It might be further postulated that witness events, occurring distinctly, one at a time between pairs of witnesses during the breaking process, each yield a third, witness obstructed species, that serves to escape fatally oriented witness events; each of the seven total species, five bases associated with the nucleic acids, and two kinds of nucleic acids are the consequence of separate but overlapping events occurring within a quickly happening event. Emergences from the same unique energy path and occurring within comparable temporal periods are postulated to possess a quality of mutual familiarity with respect to one another as explanation for processes. The energy possessing zipper quality of DNA, RNA strands are postulated to have emerged as dynamic, diversity bearing components with a longer temporal breaking range beyond that of the contemporary cytoplasm that's acts to separate the cell from its' even lesser temporal range bearing exterior to produce at the root of the life processes familiarity based perspective dependence as the criteria for the existence of the spaces they occupy.

Since the numbers that describe diversity in molecular elements are small (five bases and two species without or without uracil), greater than or equal to one (witnessed to the molecular symmetry of the uracil molecule), this account of diversity may be elemental and a precursor to this situation may not exist. It is possible to speculate that net observed overt diversity evolved to witnessible agents in the biosphere is in the range of very small numbers. The consequence of the emergence of physical obstruction at all levels to further punctuate by abbreviation a maximally open straight line, or a common preceding vast temporal period, to produce familiarity necessary for the intercourse of elements, might be speculated to acquire, in ratio to beginning points, limited and discrete diversity producing temporal increments. Diversity with respect to properties like bilateral symmetry, possession and number of appendages, cognitive and perceptual abilities, means of locomotion, or the entire of nature, despite the great varieties, combinations or complexities observed in nature, in effective comparative study may be very simple to involve in the range of one's and two's, numerical increments to describe diversities observed throughout the biosphere. 
Evidence that uracil cannot be synthesized in the laboratory from its symmetrical halves support notions that it may have a special energy arrangement and path of emergence. Uracil in DNA appears to be identified and extracted by uracil DNA glycosylase from false placement in DNA, not by recognition of its structure but by a torque that identifies its' presence and produces a physical distortion of the helix that also appears to fund energetically the excision process (Hopfner et.al., 2000, Parikh et.al, 2000). Maintenance of the cell, in addition to the existence of divides, appears to be funded energetically as if it was dictated by obstruction, physical form, shape, rather than by more complex identification processes. Additional example related to notions of obstruction and physical reality that is defined by innate properties of space, is found in a discussion of bacterial cell wall genetics and composition: analytical assessments by Danchin (Danchin et al., 2000) relate spatial constraints necessary to account for the physical structure of the cell wall from constraints related to the arrangement and composition of the gene sequences responsible to encode for its' constituents that are inherited to the structure of the cell wall. In the present discussion, the shape of the common egg (Figure 3) suggested to be the longest living form, derived from the maximally open straight line, is proposed to attain its' structure from a constraint necessary to the existence of dimension, of space, in which a familiarity of matter and energy is engendered as an atemporal shape that imposes factors of witnessibility as the consequence of a compatibility of distances of motion of matter and distances spanned by light in the conversion of energy to matter.

In this model, as in Proclus' discussion of Euclid, and in all aspects of topics related in discussion to it, the (unwitnessible) conceptual precedes the witnessible physical as a necessary criteria for the understanding and application to nature. A delineation of phenomenon of inside verses outside as they relate to the existence of witness as a function of container and contained, other than the assumed contents of space as time and distance, serves as a road sign in the path of the acquisition of understanding of processes that are imagined to have possibly been confusing in Ancient times in which exploration of the world had hardly progressed. Birth and death, imagined to demarcate all path other than the path of the individual, as road signs also, become reversed in order from the first perspective, to produce perspectives that exceed the range of possible self witness.

\section{Conclusion}

Path and the knowledge of it are often not so distinct; numerical absolutes, constants amenable to measurement are not feasibly construed to be parts of nature. The universe is considered as a heterogeneous entity of the containing and contained physical and conceptual, and its possession of the property of parametric path at all loci.

A model involving form rather than chains of cause and effect is given. When considered in analogy to the Mathematics of Euclid a striking parallel appears with established molecular mechanisms in genetics; the addition of a defining motion to the circle based on contemporary elaborated mechanics of energy metabolism to the circle that is confined philosophically to criteria of divisibility and finiteness as an abbreviated infinity, yields a geometry that resembles visually the rolling along a surface by finite in size, heterogeneous molecular complexes and motions involved in the replication, transcription and translation apparatuses of the cell. 
Visually accommodated also in the same model are emergence processes related to cultural evolution and the emergence of a much greater variety of cultural traits than composes cellular nucleic acids; cultural evolution occurs in bigger spaces and is simply a function of available space, inclination and disposition based on learning that is contoured and evolves according to preceding evolutions of obstruction to path to discrete entities sharing a common temporal element. The acquisition of knowledge, necessarily associated with a continuity of identity and entity-environment interactions, economic aspects, related to the conversion of acquired conceptual assets to material assets and visa-versa, are referred in discussion to the same model.

Though the only obvious relation to the empirical in the presented example is to the biological egg, it is argued to be a single, unique, indivisible, hence sufficient example of the consequence of the application of a motion of construction to the indivisible point, to augment understanding involving the logical containment of concepts of structure and function, of path and knowledge, 'the circumstance' within a broader philosophical realization. Paramount to new interpretation is an association of form to energy as an addition to current conceptualizations involving the association of energy to form. Dynamically occurring synergies of energy fatelessly bound to an infiniteness or null of shape and size, with its' products the witnessible, the unwitnessed, and unwitnessible, are the sources emerged form, evolved concepts and origin of diffuse in nature conceptual ability as a second olfaction; abbreviated witness as an obstruction of death precludes to emerged partnerships criteria underlined with numbers, but only criteria underlined with relative shape and size. If familiarity is held as the criteria for all intercourses, the interconvertability of energy and matter presupposes a descriptive and numberless familiarity of one with the other; if matter possesses form, form is also entailed to energy.

Paradox surviving this account, perennially entailing an order to events in the process of achieving self definition, involves the possibility of the destruction of energy, the existence of a universal ground state, and acquisition of zero potential as a consequence of multiple self witness; surviving accounts is a paradox involving the witnessible physical products of temporal unorderability, the chicken and the egg. It would seem logical to assume that ground potential is relative to location; temporal ordering of location and potential does not seem philosophically feasible, in order for zero potential to exist, location, the product of potential, must also exist. The possibility cannot be excluded of a caused loss of diversity to the distributions of heat energy as the result of application originating from confused conceptualization of road signs with the road itself, false direction that arises from falsely construed elements of containment, the contained verses the container. The possibility exists that human activities can cause naturally arrived factors of proximity to be reduced from original naturally found temporal differentials that consequent the amounts of energy contained to form; to abbreviate planarly based on planar abstraction, an apriorily abbreviated infinity.

It is shape rendered from the emergence of obstruction to path, the consequence of natural seeking that underlines all existence,. A focus is suggested that is oriented away from accounts involving sensual, perceptual witness that entails temporal chains of cause and effect, towards the elaboration of physical shape as it relates to the spaces of life and the environment. 


\section{References}

Alboussière' Thierry, Deguen' Renaud, and Melzani' Mickaël, Melting-induced stratification above the Earth's inner core due to convective translation, Nature 466, 744 -747 (5 August 2010).

Becker, Thorsten Fine-Scale Modeling of Global Plate Tectonics, Science 27 August 2010: 1020-1021.

Bourdieu, Pierre, Outline of a Theory of Practice Richard Nice trans, Cambridge University Press, NewYork 1977

Carvalho, Claudia, M.B, Zhang, Feng, James, R. Genomic disorders: A window into human gene and genome evolution, PNAS January 26, 2010 vol. 107 no. suppl 1 1765-1771.

Clifford, James, Partial Truths in Erickson, Paul, A. and Murphy, Liam, D. eds. (2006) Readings for a History of Anthropological Theory second edition pages 422-444 Broadview Press Ontario Canada, 1922.

Danchin A., Guerdoux-Jamet P., Moszer I. , and Nitschké P. Mapping the bacterial cell architecture into the chromosome, Philos Trans R Soc Lond B Biol Sci v.355(1394); Feb 29, 2000 .

Einstein, Albert Physics and Reality Journal of the Franklin Institute 221:3 359-382March 1986.

Fortes, Meyer, Evans-Pritchard, Edward Evan, (1940) Introduction to African political Systems, in Erickson, Paul, A., Murphy, Liam. D., (2006) Readings for a History of Anthropological Theory second edition Broadview Press Ontario, Canada.

Foucault, Michel, Disciple and Punishment :The Birth of the Prison, Alan Sheridan trans, Vintage Books New York 1977.

Fulconis, R. Mechanism ofRec-A Mediated Homologous Recombination Revisited By Single Molecule Nanomanipulation The EMBO Journal 25:4293-4304 September 2006.

Geertz, Clifford, Thick Description: Towards an Interpretative Theory of Culture, 1973b in Reading for a History of Anthropological Theory, Erickson, Paul, A., and Murphy, Liam, D.,pp. 318-336 eds, Ontario, Canada , 2006.

Greer, Robert. Mapping Postmodernism. Downers Grove: InterVarsity Press, 2003.

Hopfner, K.P., Parikh, S.S., and Tainer, J.A. Envisioning the Fourth Dimension of the 
Genetic Code: The Structural Biology of Macromolecular Recognition and Conformational Switching in DNA Repair Cold Spring Harb Symp Quant Biol 200065 (0) 113-126.

Harris, Marvin, (1979) The Epistemology of Cultural Materialism in Reading for a History of Anthropological Theory, Erickson, Paul, A., and Murphy, Liam, D., pp. 278-288 eds, Ontario, Canada, 2006.

Johanson, Donald, Origins of Modern Humans: Multi-regional or Out of Africa?2001, American Institute of Biological Sciences http://www.actionbioscience.org/evolution/johanson.html

Karnap R. (1956). "The Methodological Character of Theoretical Concepts" in Minnesota Studies in the Philosophy of Science, vol. I, ed. by H. Feigl and M. Scriven, Minneapolis : University of Minnesota Press.

Kirsh, Marvin, E. Induction, Space and Positive Ethics, Ludus Vitalis, vol. XVI, num. 30, pp.225-228. 2008 available on line http://ludusvitalis.org/textos/foro/30_kirsh.pdf .

Kirsh, Marvin, E. Anthropology and Parallelism : The Individual as a Universal International Journal of Sociology and Anthropology Vol. 1(7) pp. 112-115, November, 2009 Available online http://academicjournals.org/IJSA/PDF/Pdf2009/November/Kirsh.pdf.

Kirsh, Marvin, E., "Uniqueness, Self belonging and Intercourse in Nature" Lambert Academic Press 2010. ISBN-10: 3838367375, ISBN-13: 978-3838367378 preface available on line: http://ssrn.com/abstract=1647222

Latsch, Gunter Are Genetically Engineered Crops and Industrial Agriculture Killing Off the Bees? Organic Consumers Association, Finland MN, March 222007 http://www.organicconsumers.org/articles/article_4596.cfm.

Marcus, George, E. and Fischer, Michael, M.J. Anthropology as Cultural Critique 2nd edition The University of Chicago Press Chicago Illinois 1999.

Morrow, Glenn, R., (transl) PROCLUS A Commentary on the First Book of Euclid's Elements, Princeton University Press, 1970.

1970a p217 279

$1970 \mathrm{~b}$ p87 107

1970c p98 121

1970d p93 116

1970 e p94 117 
Parikh, S., S., Walcher, G., Jones, G.D. Slupphaug, G., Krokans, H., E., Blackburn, G.M., Uracil-DNA glycosylase-DNA substrate and product structures: Conformational strain promotes catalytic efficiency by coupled stereoelectronic effects PNAS May 9, 2000 vol. 97 no. $105083-5088$

Roach, John, Cannibalism Normal For Early Humans, National Geographic News April 102003 on line http://news.nationalgeographic.com/news/2003/04/0410_030410_cannibal.html.

Sacks, Karen (1982) Sisters and Wives University of Illinois press Urbana and Chicago, Illinois.

Seoighe, Cathal et al, (2000) Prevalence of Small Inversions in Yeast Gene order Evolution, Proc Natl Acad Sci U S A. 2000 December 19; 97(26): 14433-14437

Strathern, Marilyn, The Gender of the Gift, University of California Press Ltd., Los Angeles, California USA 1990.

Tartaglia, James, Rorty and the Mirror of Nature, Routledge Francis and Taylor Group, New York N.Y. 2007.

Tucker, Robert C. Ed. 1978 The Marx-Engels Reader W. W. Norton Inc. New York, NY 10110.

Weber, Max, The Protestant Ethic and the Spirit of Capitalism, translated by Talcott Parsons with an Introduction by Anthony Giddens Routledge New York, N.Y. 2005. 